\title{
OPEN A unifying model to estimate thermal tolerance limits in ectotherms across static, dynamic and fluctuating exposures to thermal stress
}

\author{
Lisa Bjerregaard Jørgensen ${ }^{\bowtie}$, Hans Malte, Michael Ørsted, Nikolaj Andreasen Klahn \& \\ Johannes Overgaard
}

Temperature tolerance is critical for defining the fundamental niche of ectotherms and researchers classically use either static (exposure to a constant temperature) or dynamic (ramping temperature) assays to assess tolerance. The use of different methods complicates comparison between studies and here we present a mathematical model (and $R$-scripts) to reconcile thermal tolerance measures obtained from static and dynamic assays. Our model uses input data from several static or dynamic experiments and is based on the well-supported assumption that thermal injury accumulation rate increases exponentially with temperature (known as a thermal death time curve). The model also assumes thermal stress at different temperatures to be additive and using experiments with Drosophila melanogaster, we validate these central assumptions by demonstrating that heat injury attained at different heat stress intensities and durations is additive. In a separate experiment we demonstrate that our model can accurately describe injury accumulation during fluctuating temperature stress and further we validate the model by successfully converting literature data of ectotherm heat tolerance (both static and dynamic assays) to a single, comparable metric (the temperature tolerated for $1 \mathrm{~h}$ ). The model presented here has many promising applications for the analysis of ectotherm thermal tolerance and we also discuss potential pitfalls that should be considered and avoided using this model.

Tolerance to temperature extremes is arguably among the most important traits defining the fundamental niche of ectotherms ${ }^{1-5}$. However, studies of thermal tolerance are difficult to compare due to the plethora of static (constant temperature) and dynamic (ramping temperature) assays used to assess tolerance limits ${ }^{3,6-19}$. Furthermore, it is difficult to relate laboratory measures of thermal tolerance to the temperature stress experienced under natural conditions particularly as the duration and intensity of temperature stress may fluctuate unpredictably.

Static and dynamic methods differ in their temperature protocol (constant and changing temperature, respectively) but also in duration of the stress during testing (see discussions in ${ }^{9,13,15,20}$ ). A solution to unify many of these different tests can be found in the exponential relation between tolerance time and temperature (see $e^{13,15,16}$ and "Discussion" below). Thus, a linear relation between $\log _{10}$ (tolerance time) and temperature in ectothermic organisms has been known for more than a century $y^{6,13,14,16,21-26}$. This analysis of thermal tolerance has also recently been revisited as a "thermal tolerance landscape" (TTL) ${ }^{15,20}$. A TTL describes the temperature-time interaction of tolerance, along with information of percentwise population mortality level ${ }^{20}$, and thus for each mortality level (for example 50\%) there is a specific temperature-time interaction, which is termed a thermal death time (TDT) curve ${ }^{6}$. Accordingly, multiple TDT curves which describes different mortality levels can be joined to form the thermal tolerance landscape for a population ${ }^{20}$. In this study, we expand this TDT curve analysis further and present a theoretical and mathematical framework that allows researchers to directly compare thermal tolerance measurements obtained during constant and dynamic experiments. Specifically, this model (and associated $R$-scripts) allows researchers to convert assessments of tolerance from static to dynamic assays 
(and vice versa) and to use lab measurements of tolerance to assess the severity of thermal stress experienced under temperature fluctuations.

\section{Theoretical foundation}

Assessment of thermal tolerance uses various endpoints (loss of righting response, onset of spasms, coma, death ${ }^{27,28}$ ), but for a given endpoint used, the estimate of tolerance will depend critically on the duration and intensity of stress exposure. The thermal death time curve usually employs death as the tolerance assessment endpoint (as per the name), but here we use the time of coma onset, a different endpoint that is however closely related to mortality at high temperature ${ }^{29-31}$. The model is likely also applicable to other endpoints and to measures of cold tolerance. In static measurements, thermal tolerance can be recorded as the duration until onset of coma $\left(\mathrm{t}_{\text {coma }}\right)$ (Fig. 1A). The TDT curve describes thermal tolerance of a species/population using the slope of the relation between assay temperature and $\log _{10}\left(\mathrm{t}_{\text {coma }}\right)$ and a point on the line (here we use $\mathrm{sCT}_{\max (1 \mathrm{~h})}$ which is the temperature causing heat coma after a 1-h exposure) (Fig. 1B). The slope represents a thermal sensitivity factor that describes the temperature change resulting in a one order of magnitude change in $t_{\text {coma }}$. To be consistent with TDT curve terminology the slope is described by the parameter $z$ where $z=-1 /$ slope $\left(\mathrm{cf}^{20}\right)$, which is analogous to $\mathrm{Q}_{10}$ where $\mathrm{Q}_{10}=10^{10 / z}$. The TDT parameters from the linear regression can be used to estimate the exposure duration $\left(\mathrm{t}_{\text {coma }}\right)$ tolerated at a specific temperature or to calculate the maximal static temperature $\left(\mathrm{sCT}_{\max }\right)$ that can be tolerated for a specific duration (but see below and associated $R$-script for details).

Another perspective of TDT curve analysis is to consider temperature as the determinant of an injury accumulation rate (Fig. 2Aii) ${ }^{13-17,23}$. From this perspective injury accumulation rate increases exponentially with temperature and the endpoint is then reached when the animal has accumulated the critical amount of injury (100\%), illustrated graphically as the area under the curve in Fig. 2. The use of coma onset as the tolerance endpoint means that this "injury accumulation rate" is actually a "rate of failure induction", but since heat coma and mortality is closely associated in time, we have opted to use the term injury accumulation rate. Applying the concept of injury accumulation rates, it is possible to use TDT parameters to find the tolerance limits using other combinations of static, dynamic or fluctuating stress exposure. To validate this model empirically, we use Drosophila melanogaster to parameterize a TDT curve with static experiments and then use the TDT parameters to predict (and test) the accumulated injury from two sequential stress exposures at two different temperatures (as in Fig. 2C) and to predict (and test) heat failure in flies exposed to randomly fluctuating temperatures (as in Fig. 2D).

To make this method accessible to researchers we describe the mathematical foundation and provide $R$-scripts to directly derive TDT parameters and use them to assess tolerance limits. The scripts can derive TDT parameters from static data sets (where time to failure, $t_{\text {coma }}$, is measured at different constant temperatures), or from dynamic data sets (where the maximal temperature tolerated, $C T_{\max }$, is measured using different ramping rates). The scripts also allow for construction of a TDT curve using only a single static or dynamic estimate of heat tolerance, but we caution that the model will be extremely sensitive to the (untested) assumed value of $z$ that must be provided. Irrespective of the type of input data, the scripts can predict heat failure at various static and dynamic measurements from TDT parameters or integrate TDT parameters with data of temperature fluctuations to predict heat stress under fluctuating conditions. To test the applicability of the model we compare estimates of heat tolerance in nine ectotherm species from many different literature sources with the assumption that each of these species are characterised by a reasonably constant species-specific heat tolerance. Specifically, we use the original experimental data to generate TDT parameters and then estimate the temperature required to cause heat failure in $1 \mathrm{~h}$.

As with most models in biology the quality of the input data determines the quality of the model output. Furthermore, all models have boundaries and limitations for their applications. We therefore discuss important considerations including: (i) The importance of using experimental data for model parameterization within the time and temperature domain of interest, (ii) The risks of model extrapolation, and (iii) The need to consider model boundaries and to consider interactions with repair and hardening. These different considerations are important as they set the boundaries in time and temperature for the applicability of the model.

\section{Materials and methods}

Mathematical foundation. If we assume that the acute, temperature-related injury accumulation rate of an animal depends on temperature by some function $R(T)$ then the amount of accumulated injury/damage $(d)$ at some time $t$ is given by:

$$
\mathrm{d}(\mathrm{t})=\int_{\mathrm{t}_{\mathrm{c}}}^{\mathrm{t}} \mathrm{R}[\mathrm{T}(\tau)] \mathrm{d} \tau
$$

here $T(\tau)$ is the time-varying temperature regime and $t_{c}$ is the time where injury accumulation starts. The temperature at $t_{c}$ is $T_{c}$, and up to this temperature the repair of injury can match the generation of injury. For Eq. (1) to hold true, however, we must require that once temperature surpasses $T_{c}$ it will stay above $T_{c}$. At some time $t_{L d}$ (the dynamic knockdown time, vertical arrow in Fig. 2Bi), a lethal dose $d_{L}$ (area under the curve in Fig. 2Bii) has been attained and the animal is knocked down. If injury generation rate depends exponentially on temperature, and if injury repair rate is maximized at $T_{c}$ (reached at $t_{c}$ ), then the accumulated lethal dose is related to the knockdown time $t_{L d}$ by:

$$
d_{L}=R_{0} \int_{t_{c}}^{t_{L d}}\left(e^{k\left(T(t)-T_{c}\right)}-1\right) d t
$$



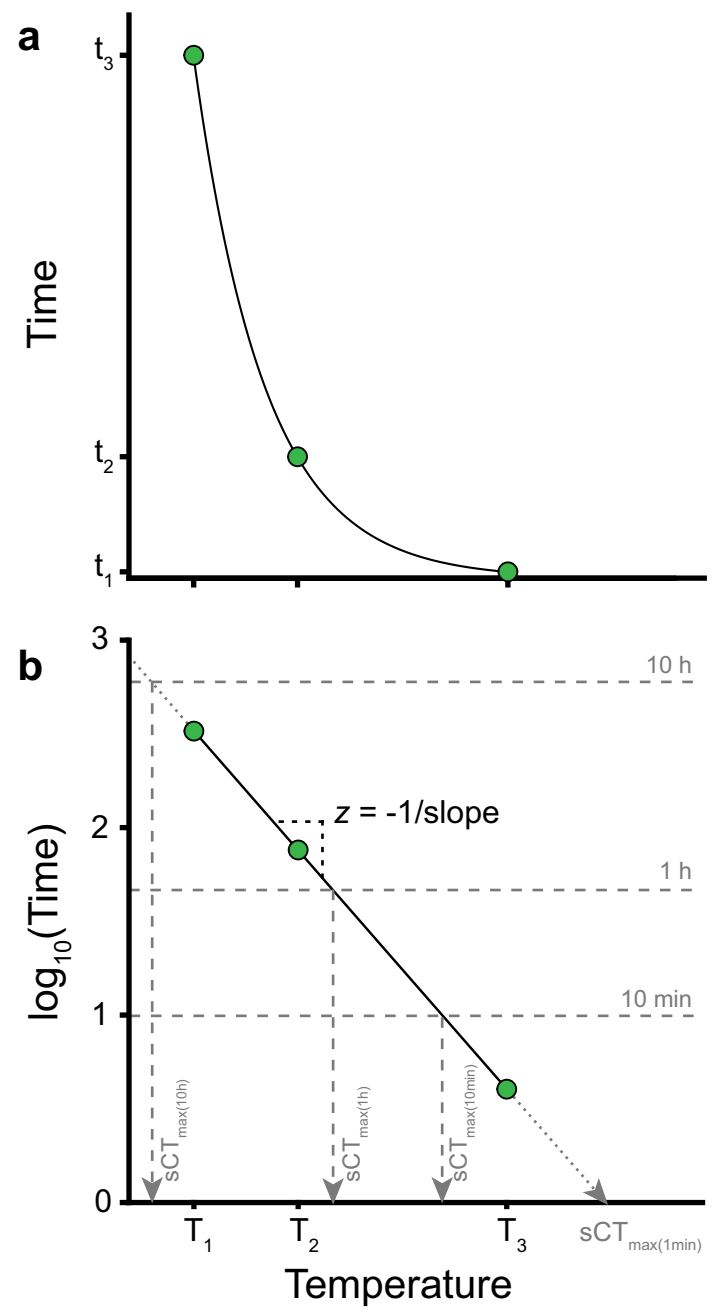

Figure 1. Exponential relation between temperature and $t_{\text {coma }}$ presented as a thermal death time (TDT) curve. (A) Time to coma $\left(t_{\text {coma }}\right)$ decreases exponentially with temperature. (B) Observations from (A) presented as a TDT curve (however with coma rather than death time) where the linearity of $\log _{10}$-transformed $t_{\text {coma }}$ versus temperature indicates the exponential relation (full line). The negative inverse slope of the TDT curve is used as the thermal sensitivity parameter $z\left(z=\right.$ the change in temperature required to change $t_{\text {coma }}$ by an order of magnitude). Intersections between the TDT curve and horizontal lines show the temperature $\left(\mathrm{sCT}_{\max }\right)$ at which coma is expected to occur after a fixed time (e.g. $\left.1 \mathrm{~h}, \mathrm{sCT}_{\max (1 \mathrm{~h})}\right)$. Extrapolations in either end of the TDT curve (dotted lines) are tempting but should be met with caution as the relationship is only linear in a certain timetemperature interval (see "Discussion" section).

Due to the potent (high $k$ ) relation between injury accumulation rate and temperature, and because the true $T_{c}$ for any species is rarely known, we simplified this to (see Fig. $\mathrm{S} 1$ for further justification):

$$
\mathrm{d}_{\mathrm{L}} \approx \mathrm{R}_{0} \int_{\mathrm{t}_{\mathrm{c} *}}^{\mathrm{t}_{\mathrm{Ld}}} e^{\mathrm{k}\left(\mathrm{T}(\mathrm{t})-\mathrm{T}_{\mathrm{c} *}\right)} \mathrm{dt}
$$

where $t_{c^{*}}$ is some start time for integration where the temperature $T$ attains some convenient value $T_{c^{*}}$ (e.g. rearing temperature) below the true $T_{c}$. In the event that exposure temperature is constant (and above $T_{c^{*}}$ ) we get:

$$
\mathrm{d}_{\mathrm{L}}=\mathrm{t}_{\mathrm{Ls}} \mathrm{R}_{0} \mathrm{e}^{\mathrm{k}\left(\mathrm{T}-\mathrm{T}_{\mathrm{c} *}\right)}
$$

where $t_{L s}$ is the static knockdown time. Rearranging this leads to an exponential relation between the static knockdown time and temperature, which is here called sCT $\mathrm{m}_{\max }\left(\right.$ static $\left.\mathrm{CT}_{\max }\right)$ :

$$
\mathrm{t}_{\mathrm{Ls}}=\alpha \mathrm{e}^{-\mathrm{k}\left(\mathrm{sCT}_{\max }-\mathrm{T}_{\mathrm{c*}}\right)}
$$

where $\alpha=d_{L} / R_{0}$. This is identical to the usual form of the TDT curve if $\log (\alpha)=s C T_{\max } / z$ and $k=\ln (10) / z$.

Assuming that the accumulated injury is always the same at knockdown then, once knowing knockdown time and temperature in one experimental setting, will allow calculation of expected knockdown temperatures and/or times in other experimental settings. Thus, in the case of a linearly increasing temperature ramp of the 


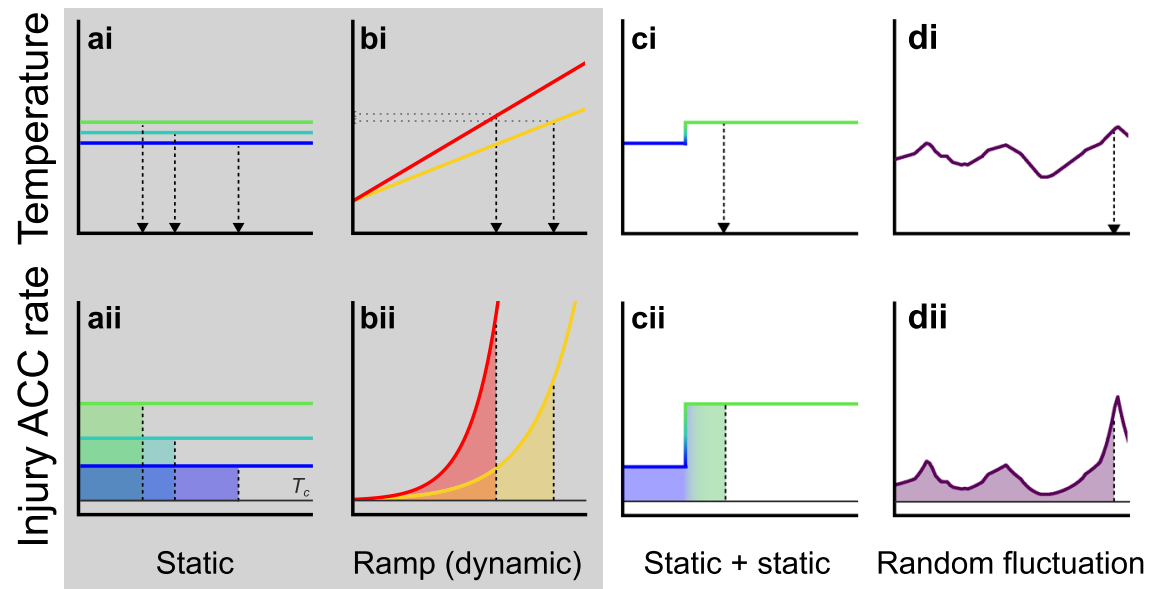

Figure 2. Temperature profiles of heat tolerance experiments and their corresponding temperature-dependent injury accumulation (ACC) rates based on parameters from the thermal death time curve. $\mathrm{x}$-axes represent a time scale. Shaded areas under the curves represent identical amounts of accumulated injury that result in heat failure (lethal dose, Aii-Dii). The exposure duration resulting in accumulation of the lethal dose is reported as $\mathrm{t}_{\text {coma }}$ (static) or $\mathrm{CT}_{\max }$ (dynamic), marked by vertical and horizontal arrows, respectively, in Ai-Di. The grey box highlights the two classical assay types (static and dynamic). Note that injury only accumulates at temperatures above a specific critical temperature $\left(\mathrm{T}_{c}\right.$, thin black line). (Ai-Aii) Exposure to a static temperature yields a constant temperature-dependent injury accumulation rate. (Bi-Bii) Exposure to a dynamic ramp where temperature changes by a fixed rate resulting in an exponentially increasing injury accumulation rate with time. The fast ramping rate (red) results in a shorter exposure before $\mathrm{CT}_{\max }$ is observed than the slow rate (yellow), but as the injury accumulation rate increases more slowly for the low ramping rate, the final $\mathrm{CT}_{\max }$ is lower. (Ci-Cii) During initial exposure to a static temperature (blue), some injury is accumulated, but not sufficient to trigger coma onset. Successive exposure to another static temperature (green) produces the critical amount of injury, but as some injury had already accumulated, the exposure to the second temperature is shorter than if only this temperature had been used (compare with Aii). The prediction of the model will only hold true if injury is additive at the two static temperatures. (Di-Dii) Varying (natural or laboratory generated) temperature changes that cannot be described by a simple fixed ramping rate. However, knowing the temperature-time profile permits calculations of the injury accumulation at any time and thus estimation of $\mathrm{t}_{\text {coma. }}$.

form $T(t)=T_{0}+b^{\bullet} t$, where $T_{0}$ is the ramp start temperature and $b$ is the ramping rate, the dynamic knockdown time and temperatures can be calculated by: (- note that in the original paper ${ }^{15}$ the " $l n$ " and the $k$ in front of $\left(T_{c^{*}}-T_{0}\right)$ was lost in typesetting):

$$
\begin{aligned}
\mathrm{t}_{\mathrm{Ld}} & =\frac{1}{\mathrm{~kb}} \ln \left[\mathrm{kbt}_{\mathrm{Ls}} \mathrm{e}^{\mathrm{k}\left(\mathrm{sC} \mathrm{T}_{\max }-\mathrm{T}_{0}\right)}+\mathrm{e}^{\mathrm{k}\left(\mathrm{T}_{\mathrm{c} *}-\mathrm{T}_{0}\right)}\right] \\
\mathrm{dCT}_{\max } & =\mathrm{T}_{0}+\frac{1}{\mathrm{k}} \ln \left[\mathrm{kbt}_{\mathrm{Ls}} \mathrm{e}^{\mathrm{k}\left(\mathrm{sCT}_{\max }-\mathrm{T}_{0}\right)}+\mathrm{e}^{\mathrm{k}\left(\mathrm{T}_{\mathrm{c} *}-\mathrm{T}_{0}\right)}\right] .
\end{aligned}
$$

One can also express $s C T_{\max }$ as a function of $d C T_{\max }$ :

$$
\mathrm{sCT}_{\max }=\mathrm{T}_{0}+\frac{1}{\mathrm{k}} \ln \left[\frac{1}{\mathrm{kbt}_{\mathrm{Ls}}}\left(\mathrm{e}^{\mathrm{k}\left(\mathrm{dCT}_{\max }-\mathrm{T}_{0}\right)}-\mathrm{e}^{\mathrm{k}\left(\mathrm{T}_{\mathrm{c} *}-\mathrm{T}_{0}\right)}\right)\right] .
$$

If the temperature varies randomly (and therefore is not monotonically increasing) it is no longer possible to calculate an expected knockdown temperature from knockdown temperatures obtained in static or dynamic ramp experiments. However, the expected knockdown time can still be found by evaluating (usually numerically) the integral of Eq. (3) as a function of time.

Using the equations. We provide two R-scripts (https://github.com/MOersted/Thermal-tolerances, see guide in Supplementary Information) to aid TDT parameterization from static and dynamic assays. One script establishes the TDT parameters from the knockdown time at two or more static temperatures (Fig. 1), while the other script derives TDT parameters from two or more values of $d C T_{\max }$ obtained by different ramping rates and/or start temperatures (Fig. 2B). Using dynamic input data, $s C T_{\max }$ and $z$ is estimated either through nonlinear curve fitting in case $d C T_{\max }$ is available for three or more ramping rates, or by solving two equations (either 7a or 7b) with two unknowns when $d C T_{\max }$ is known for two ramping rates (see Supplementary Information for details).

Knowing only one $\mathrm{dCT}_{\max }$ or $\mathrm{sCT}_{\max }$ will not suffice to establish a TDT curve since $k$ (and thus $z$ ) in Eqs. (7a) and (7b) will be left unknown. In such cases, an estimated value of $z$ must be supplied (some guidance is provided 
in Supplementary Information, Table S1), to allow the establishment of a crude TDT curve, which will be subject to uncertainty and sensitive to extrapolation (see "Discussion" section).

Once the TDT curve is parameterized, both scripts have four available outputs; (i) estimated exposure duration $\left(\mathrm{t}_{\text {coma }}\right)$ tolerated at specific temperatures, (ii) estimated static temperature $\left(s C T_{\max }\right)$ that can be tolerated for specific durations, (iii) estimated maximal dynamic temperature $d C T_{\max }$ that can be tolerated in experiments with specific ramp rates, and (iv) estimated injury accumulation under fluctuating temperatures (Fig. 2D), and thus the exposure duration tolerated before a given percent of lethal injury has accumulated.

Empirical experiments for model validation. Animal husbandry. Drosophila melanogaster (collected in Denmark, 2011) were kept in bottles with oat-based Leeds medium ${ }^{11}$ ( $19^{\circ} \mathrm{C}$, constant light). Experimental flies were produced by transferring a spoonful media containing egg/larvae to fresh bottles $\left(23^{\circ} \mathrm{C}, 14: 10 \mathrm{~L}: \mathrm{D}\right.$ cycle). Emerging flies were collected every two days and after 2-5 days flies were briefly anaesthetised with $\mathrm{CO}_{2}$ $(<5 \mathrm{~min})$, then separated by sex and returned to fresh vials. Flies were allowed $>2$ days to recover from anaesthesia before experiments ${ }^{32}$, resulting in 4-8 days old experimental flies.

Thermal death time (TDT) curves. TDT curves were generated by exposing flies to different stressful static temperatures and measuring the time to coma ( $\mathrm{t}_{\text {coma }}$, like Fig. 1). Experiments were conducted at temperatures resulting in heat coma onset ranging from $\sim 10 \mathrm{~min}$ to $5 \mathrm{~h}$ using the experimental setup described in ${ }^{15}$. Briefly, flies were placed individually in 5-mL glass vials with a droplet of Leeds medium in the cap (food and water source), then mounted to a rack and submerged in a preheated temperature-controlled water bath. The median value of $t_{\text {coma }}$ was reported for each temperature allowing for long experiments $(>30 \mathrm{~min})$ to be terminated once more than half of the flies had entered heat coma (See ${ }^{15}$ for use of median values). A TDT curve was parameterized for each sex by linear regression of the $\log _{10}$-transformed median $t_{\text {coma }}$ against the assay temperature as described above (Fig. 1).

Test of injury additivity between two static temperatures. To investigate whether heat injury acquired at different temperatures is additive, flies were exposed to one stressful temperature $\left(T_{1}\right)$ and successively transferred to another stressful temperature $\left(\mathrm{T}_{2}\right)$ (Fig. $2 \mathrm{C}$ ). The two temperatures were selected from the TDT curve: a 'low' temperature $\left(\sim 36.5^{\circ} \mathrm{C}\right)$ with expected $\mathrm{t}_{\text {coma }}>4 \mathrm{~h}$ and a 'high' temperature $\left(\sim 39.5^{\circ} \mathrm{C}\right)$ with expected $\mathrm{t}_{\text {coma }}<1 \mathrm{~h}$. Experiments were performed with both the 'high' and 'low' temperature as $\mathrm{T}_{1}$ to examine whether additivity was independent of the heat stress intensity during the first exposure. In each experimental run, six groups $(n=10)$ of both sexes were placed at $\mathrm{T}_{1}$ and then transferred to $\mathrm{T}_{2}$ at regular intervals for recording of $\mathrm{t}_{\text {coma }}$. For each combination of sex and treatment order ('high' or 'low' temperature as $\mathrm{T}_{1}$ ) the median exposure time at $\mathrm{T}_{1}$ and $\mathrm{T}_{2}$ was recalculated as fractions of the $t_{\text {coma }}$ predicted by the TDT curve. As an example, an initial exposure of $15 \mathrm{~min}$ to the higher test temperature $\left(39.5^{\circ} \mathrm{C}\right.$, TDT curve-predicted $\left.\mathrm{t}_{\text {coma }}=50 \mathrm{~min}\right)$ corresponds to a fraction of $15 \mathrm{~min} / 50 \mathrm{~min}=0.3$ of injury resulting in coma. The hypothesis of additivity predicts that the fractions of accumulated injury resulting in coma should sum to 1 , and accordingly the predicted fraction of time to coma at $\mathrm{T}_{2}$ should be 0.7 . Thus, if the lower test temperature $\left(36.5^{\circ} \mathrm{C}\right)$ has a TDT curve-predicted median $\mathrm{t}_{\text {coma }}$ of $300 \mathrm{~min}$, a fraction of 0.7 corresponds to $300 \mathrm{~min} \cdot 0.7=210 \mathrm{~min}$.

Test of injury additivity during temperature fluctuations. Animals in the field experience temperature fluctuations. If injury is additive, it should be possible to predict $t_{\text {coma }}$ under such conditions by considering fluctuations as many additive exposures to different temperatures each characterised by an injury accumulation rate (calculated from TDT curve, Fig. 2D). To investigate whether heat injury during fluctuating temperatures can be modelled from TDT parameters, $t_{\text {coma }}$ was observed in flies exposed to randomly fluctuating temperatures and compared to a TDT curve-predicted $t_{\text {coma. }}$. Flies were divided into 13 experimental groups $(n=16-18)$ for each sex. Groups were introduced to the water bath at different times, which was randomly and sequentially programmed to heat or cool. Consequently, each group experienced a unique temperature profile (range: $34.5-$ $42.5^{\circ} \mathrm{C}$ ). The median $\mathrm{t}_{\text {coma }}$ for each sex and experimental group was compared to the $t_{\text {coma }}$ predicted by the TDT curve. Specifically, to predict $t_{\text {coma }}$ under these conditions the associated $R$-script was given the average temperature for each 10-s period and injury accumulation for each period was then calculated from the TDT curve. Injury accumulated during each 10 -s interval was summed until the critical amount of injury had accumulated resulting in predicted $t_{\text {coma }}$.

Model validation using literature data. Measures of heat tolerance from different publications are difficult to compare as they are based on different experimental procedures or conditions. To examine whether our model can improve comparison of published tolerance estimates, we collected heat tolerance measures from both static and dynamic assays for nine ectothermic species including a crustacean (Daphnia magna Straus 1820), insects (D. melanogaster Meigen 1830, Drosophila subobscura Collin 1936, Glossina pallidipes Austen 1903, Tenebrio molitor L. 1758), collembolans (Folsomia candida Willem 1902, Orchesella cincta L. 1758) and fishes (Gambusia affinis Baird \& Girard 1853, Salmo salar L. 1758). Literature search was aided by an overview of dynamic assays with at least three ramping rates ${ }^{33}$, the GlobTherm database ${ }^{34}$ and a review of $\mathrm{CT}_{\max }$ in aquatic ectotherms ${ }^{35}$. For static assays the time of failure and assay temperature were recorded while the ramping rate, $\mathrm{CT}_{\max }$ and the initial temperature $\left(\mathrm{t}_{0}\right)$ were noted for dynamic assays. Acclimation temperature was recorded if specified. The aim was to gather multiple heat tolerance measures from the same publication to provide input for TDT curves, but publications with fewer assay temperatures or ramping rates were also included to examine how the model operated using assumed values of $z$. The data set included 155 static entries and 227 dynamic entries from 55 publications. For all studies included the associated $R$-scripts were used to derive TDT param- 

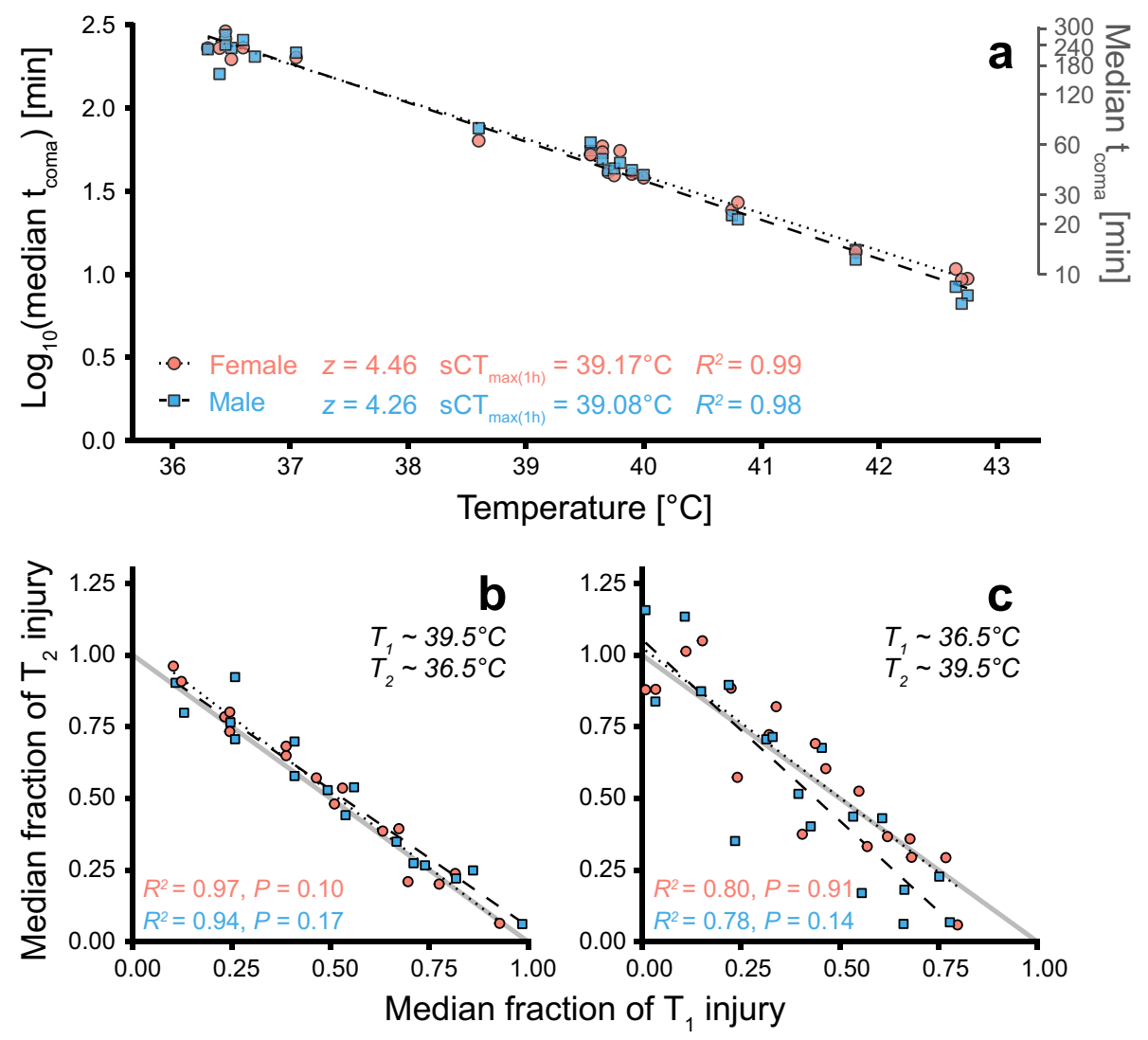

Figure 3. Thermal death time curves and test of injury additivity between two temperatures. (A) Flies were exposed to a static stressful temperature until coma onset $\left(\mathrm{t}_{\text {coma }}\right)$. Each point is the median $\mathrm{t}_{\text {coma }}$ of a group $(n=2-10)$ and TDT curves are made for each sex by linear regressions of median $\log _{10}\left(\mathrm{t}_{\text {coma }}\right)$ vs. assay temperature. (B) Groups of flies $\left(\mathrm{n}=5-6\right.$ per sex) were exposed to a high temperature $\left(\sim 39.5^{\circ} \mathrm{C}\right)$ and then, after different durations, transferred to a lower temperature $\left(\sim 36.5^{\circ} \mathrm{C}\right)$ where median $\mathrm{t}_{\text {coma }}$ was recorded. The exposures are shown as fractions of injury resulting in coma at $\mathrm{T}_{1}$ (x-axis) and $\mathrm{T}_{2}$ (y-axis), where small and large fractions indicate brief and long exposures, respectively (i.e. brief exposure results in small accumulation of injury relative to the critical amount of injury). If injury acquired at two different temperatures is additive, the two fractions should sum to 1, i.e. follow the (full) line of additivity. (C) A similar experiment as (B), but with initial exposure to $\sim 36.5^{\circ} \mathrm{C}$ and the subsequent exposure to $\sim 39.5^{\circ} \mathrm{C}$.

eters to estimate the static temperature that causes heat failure after a 1-h exposure $\left(\mathrm{sCT}_{\max (1 \mathrm{~h})}\right)$. For studies with single tolerance measures we assumed the mean species value of $z$ calculated from the other publications in our analysis (Table S1).

\section{Results}

Thermal death time curves. Median time to heat coma $\left(\mathrm{t}_{\text {coma }}\right)$ ranged from 7 to $289 \mathrm{~min}(\sim 5 \mathrm{~h})$ in flies exposed to constant, stressful temperatures ranging $36.30-42.75^{\circ} \mathrm{C}$ (Fig. $3 \mathrm{~A}$ ). The resulting TDT curves were well-fitted $\left(R^{2}>0.98\right)$ for both sexes, showing that the exponential function is appropriate to describe the negative relation between temperature and $\mathrm{t}_{\text {coma }}$. These TDT parameters $\left(z\right.$ and $\left.\mathrm{sCT}_{\max (1 \mathrm{~h})}\right)$ were used to characterise temperature-specific injury accumulation rates in subsequent experiments.

Test of injury additivity between two static temperatures. To investigate whether heat injury acquired at two stressful temperatures is additive, groups of flies were exposed to a static temperature $\left(\mathrm{T}_{1}\right)$ and subsequently exposed to another static temperature $\left(\mathrm{T}_{2}\right)$ (Fig. 3B,C). Injury accumulated at the two temperatures was scored as the fraction of injury required to enter coma at $\mathrm{T}_{1}$ and $\mathrm{T}_{2}$, respectively (calculated from TDT curves, Fig. 3A), and these fractions summed to $\sim 1$ (graphically points are scattered around the line of additivity, $R^{2}>0.78$, Fig. 3B,C). This indicates that heat injury at two static stressful temperatures is additive, which is supported statistically as the linear regressions of the fractional injury accumulation to $t_{\text {coma }}$ at $\mathrm{T}_{1} v s$. $\mathrm{T}_{2}$ were not significantly different from the line of additivity for neither sex nor temperature sequence $\left(\mathrm{F}_{(2,14-16)}<2.7, P>0.1\right.$, Fig. 3B,C, Table S2).

Test of injury additivity during random temperature fluctuations. Groups of flies were exposed to randomly fluctuating temperatures to investigate whether injury accumulation was also additive under these 

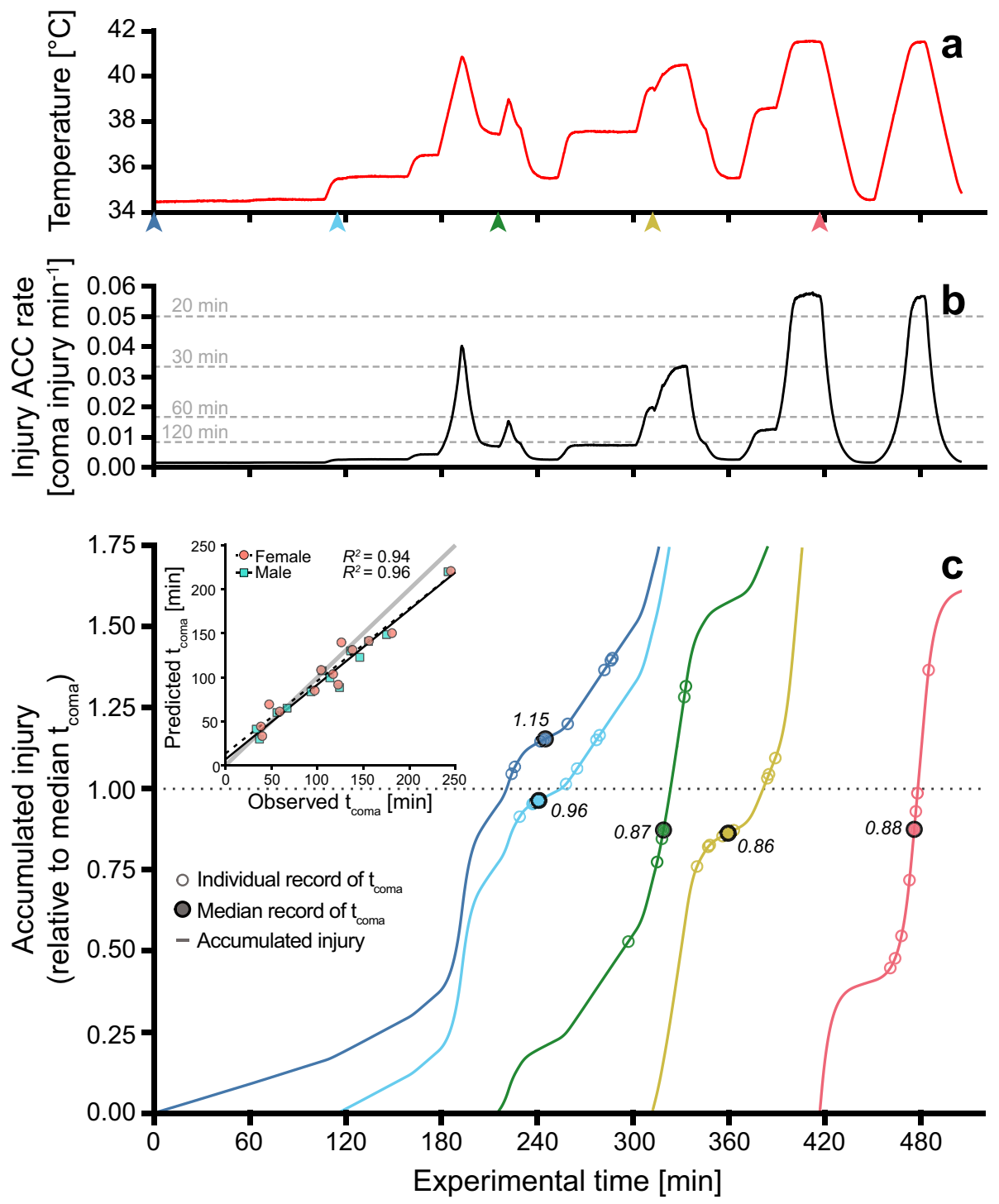

Figure 4. Test of injury additivity during random temperature fluctuations. (A) Fluctuating temperature profile from one of the experiments. (B) The corresponding temperature specific injury accumulation (ACC) rates for each 10-s interval calculated from the TDT curve (Fig. 3A). Rates are presented as a fraction of coma injury $\mathrm{min}^{-1}$ (i.e. 0.01 corresponds to an injury rate that would cause coma in $100 \mathrm{~min}$ ), and dashed lines show the injury accumulation rate that, if held constant, would result in coma after 20, 30, 60 and $120 \mathrm{~min}$. (C) Groups of flies $(n=8-9)$ were introduced at different times during the assay (arrowheads in $(\mathbf{A})$ ), and thus each group experienced a unique sequence of fluctuating temperatures. The temperature-specific injury accumulation rates were used to predict the gradual injury accumulation (coloured lines), and accordingly, when each experimental group should have accumulated the critical amount of injury according to the TDT curve (fraction =1, dotted line). Numbers accompanying median points are the calculated amount of injury accumulated at that specific time for this group. (Inset) Observed vs predicted $t_{\text {coma }}$ from all experiments (grey line of unity).

conditions (Figs. 2D, 4A). Each group experienced a unique sequence of stressful temperatures resulting in exposures spanning 33-245 min before the last fly entered coma. For each of the random temperature exposures the accumulated amount of injury was predicted using TDT parameters and the accompanying $R$-script (Fig. 4B). Median $t_{\text {coma }}$ was recorded for each sex in the 13 test groups and compared to the predicted $t_{\text {coma }}$ for each specific fluctuating temperature sequence (Fig. 4C). Predicted and observed $t_{\text {coma }}$ correlated strongly in both sexes $\left(R^{2}>0.94\right)$, and though the linear regressions deviated slightly from the line of unity $\left(\mathrm{F}_{(2,11)}>5.9\right.$; $P<0.02$, Table S3), the predicted and observed $\mathrm{t}_{\text {coma }}$ were not significantly different (inset in Fig. 4C, MannWhitney $U$ test, male: $\mathrm{W}=96, P=0.58$; female: $\mathrm{W}=88, P=0.84)$. The model calculated the mean amount of injury accumulated at the median observed $t_{\text {coma }}$ to be 1.10 (range: $0.75-1.36$ ) and 1.09 (range: $0.86-1.46$ ) for males and females, respectively, where 1.00 is the TDT curve predicted amount causing coma (Fig. 4C). 


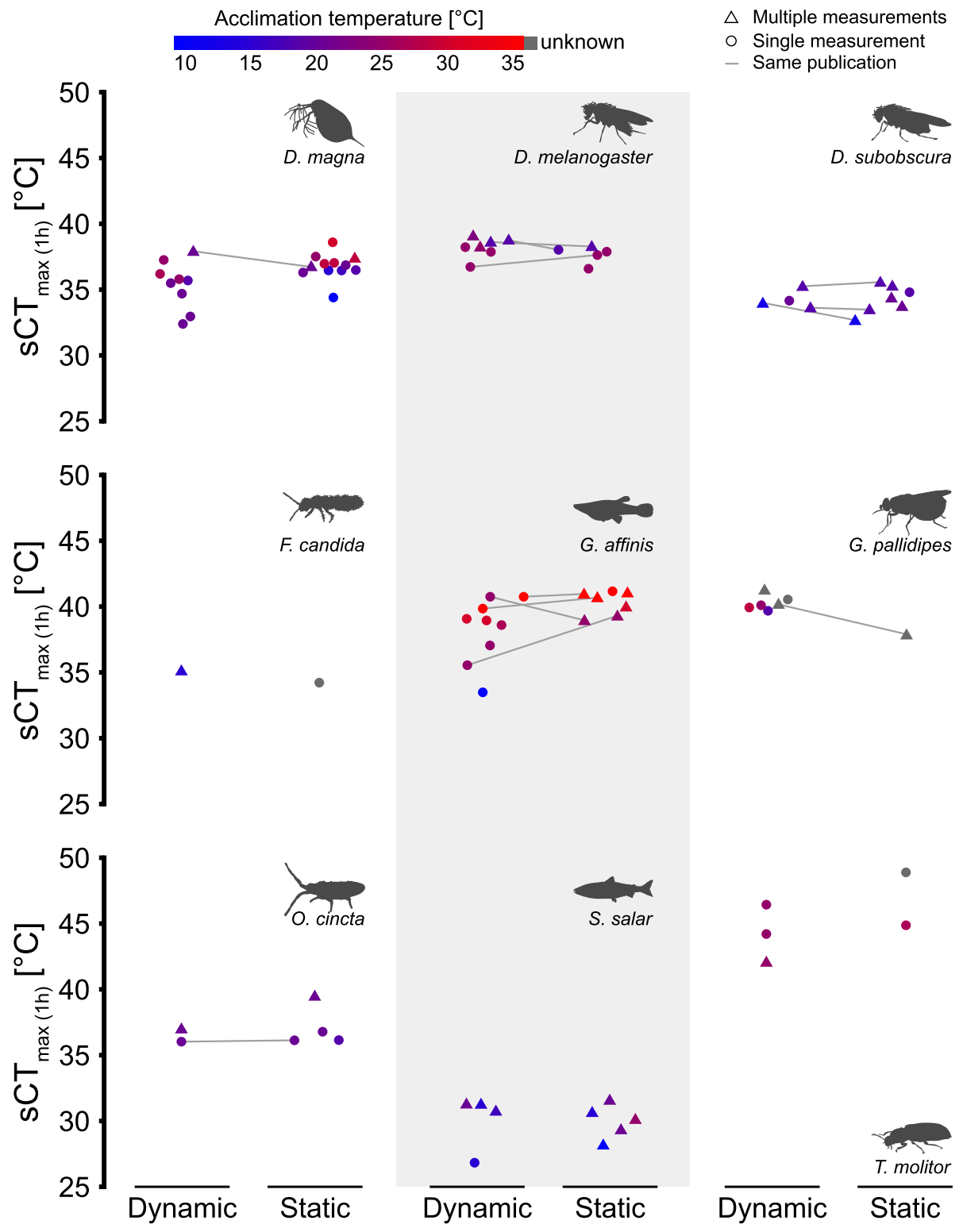

Figure 5. Heat tolerance measures from the literature re-calculated to the temperature resulting in heat failure after a 1-h exposure $\left(\mathrm{sCT}_{\max (1 \mathrm{~h})}\right)$. Heat tolerance measurements from both dynamic and static assays were obtained from the literature for nine ectothermic species and used as input for the associated $R$-scripts. If multiple measures of the same assay type were available in a publication (triangles), these measurements were

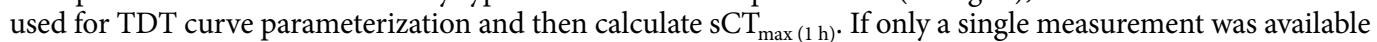
(circle) an estimated value of $z$ was supplied to create a TDT curve for calculation of $\mathrm{sCT}_{\max (1 \mathrm{~h})}(\mathrm{see}$ main text). Connected points represent publications that provided both dynamic and static measures and colour indicates acclimation temperature.

Using TDT parameters to compare heat tolerance measures from the literature. Literature values of heat tolerance were obtained for nine ectotherm species and for each publication we used values as model input to calculate a common heat tolerance estimate; the static temperature estimated to result in heat failure after a 1-h exposure $\left(\mathrm{sCT}_{\max (1 \mathrm{~h})}\right.$, Fig. 5). Input data included both dynamic and static experiments and after conversion to a common metric $\mathrm{sCT}_{\max (1 \mathrm{~h})}$ we generally found overlap within species and it is for example possible to discern large interspecific differences in heat tolerance (compare D. melanogaster and D. subobscura or G. affinis and S. salar, Fig. 5). In species with a relatively large intraspecific variation, higher acclimation temperatures tend to increase heat tolerance, although this was not formally tested. 


\section{Discussion}

Fitting tolerance time versus temperature to build a thermal death time curve. The high coefficients of determination found in the D. melanogaster TDT curves (Fig. 3A) are not uncommon and the exponential relation has consistently been found to provide a good fit of tolerance time vs. temperature in ectotherms $3,15,20,22-24$. Tolerance time vs. temperature data are also well fitted to Arrhenius plots which are based on thermodynamic principles (see for example ${ }^{15,36}$ ) and the absence of breakpoints in such plots provides a strong indication (but not direct proof) that the cause of coma/heat failure under the different intensities of acute heat stress is related to the same physiological process regardless whether failure occurs after $10 \mathrm{~min}$ or $10 \mathrm{~h}^{2,3}$ (but see "Discussion" section below). Despite the superior theoretical basis of Arrhenius analysis, we proceed with simple linear regressions of $\log _{10}$-transformed $t_{c o m a}$ (TDT curve) as this analysis likewise provides a high $R^{2}$ and is mathematically more straightforward. The physiological cause(s) of ectotherm heat failure are poorly understood $^{37,38}$ but we argue that they are founded in a common process where heat injury accumulates at a temperature-dependent rate until a species-specific critical dose is attained (area below the curve and above $\mathrm{T}_{\mathrm{c}}$ in Fig. 2). Thus, the organism has a fixed amount (dose) of thermally induced stress that it can tolerate before evoking the chosen endpoint. The experienced temperature of the animals then dictates the rate of which this stress is acquired, and accordingly when the endpoint is reached (Fig. 2) It is this reasoning that leads to TDT curves and explains why heat stress can be additive and thus also determines the boundaries of TDT curve modelling.

Injury is additive across different stressful assay temperatures. If heat stress acquired at intense and moderate stress within the span of the TDT curve acts through the same physiological mechanisms or converges to result in the same form of injury, then it is expected that injury is additive at different heat stress intensities. This hypothesis was tested by exposing flies sequentially to two static temperatures (different injury accumulation rates) and observe whether coma occurred as predicted from the summed injury (Fig. 2C). The accumulated heat injury at the two temperatures was found to be additive regardless of the order of temperature exposure (Fig. 3B,C). This finding is consistent with a conceptually similar study using speckled trout which also found strong support for additivity of heat stress at different stressful temperatures ${ }^{13}$. The exact physiological mechanism of heat injury accumulation is interesting to understand in this perspective, but it is not critical as long as the relation between temperature and injury accumulation rate is known.

If injury accumulation is additive irrespective of the order of the heat exposure, we can extend the model to fluctuating temperature conditions. We have previously done this by accurately predicting dynamic $\mathrm{CT}_{\max }$ from TDT parameters obtained from static assays for 11 Drosophila species (Fig. 6A, see "Discussion" section below and ${ }^{15}$ ). Here we extend this to temperature fluctuations that cannot be described by a simple mathematical ramp function. Specifically, groups of flies were subjected to randomly fluctuating temperatures and the observed $t_{\text {coma }}$ was then compared to $t_{\text {coma }}$ predicted using integration of heat injury based on TDT parameters (Fig. 4). The injury accumulation (Fig. 4C) was calculated by introducing the fluctuating temperature profiles in the associated $R$-script and the observed and predicted $\mathrm{t}_{\text {coma }}$ was found to correlate well $\left(R^{2}>0.94\right)$ across the 13 groups tested for each sex. These results further support the idea that injury is additive across a range of fluctuating and stressful temperatures and hence that similar physiological perturbations are in play during moderate and intense heat stress. It is important to note that in these experiments, temperatures fluctuated between 34.5 and $42.5{ }^{\circ} \mathrm{C}$ and accordingly the flies were never exposed to benign temperatures that could allow repair or hardening (see below).

In conclusion, empirical data (present study; ${ }^{6,13,14,22}$ ) support the application of TDT curves to assess heat injury accumulation under fluctuating temperature conditions both in the lab and field for vertebrate and invertebrate ectotherms. Potential applications could be assessment of injury during foraging in extreme and fluctuating environments (e.g. ants in the desert ${ }^{39}$ or lizards in exposed habitat ${ }^{40}$ ) or for other animals experiencing extreme conditions $s^{41,42}$. The associated $R$-scripts allow assessment of percent lethal damage under such conditions if the model is provided with TDT parameters and information of temperature fluctuations (but see "Discussion" section of model limitations below).

Model application for comparison of static versus dynamic data. There is little consensus on the optimal protocol to assess ectotherm thermal tolerance and many different types of static or dynamic tests have been used to assess heat tolerance. TDT curves represent a mathematical and theoretical approach to reconcile different estimates of tolerance as the derived parameters can subsequently be used to assess heat injury accumulation at different rates (temperatures) and durations $s^{13,15,16}$. Here we provide $R$-scripts that enable such reconciliation and to demonstrate the ability of the TDT curves to reconcile data from static vs. dynamic assays we used published measurements of heat tolerance for 11 Drosophila species using three dynamic and 9-17 static measurements for each species ${ }^{43}$. Introducing data from only static assays we derived TDT parameters and subsequently used these to predict dynamic $\mathrm{CT}_{\max }$ that were compared to empirically observed $\mathrm{CT}_{\max }$ for three ramp rates (Fig. 6A). In a similar analysis, TDT parameters were derived from the three dynamic (ramp) experiments to predict $t_{\text {coma }}$ at different static temperatures which were compared to empirical measures from static assays (Fig. 6B). Both analyses found good correlation between the predicted and observed values regardless whether the TDT curve was parameterized from static or dynamic experiments (Fig. 6). However, predictions from TDT curves based on three dynamic assays were characterised by more variation, particularly when used to assess tolerance time at very short or long durations. Furthermore, D. melanogaster and D. virilis which had the poorest correlation between predicted and observed $t_{\text {coma }}$ in Fig. $6 \mathrm{~B}$ had values of $z$ from the TDT curves based on dynamic input data that were considerably different from values of $z$ derived from TDT curves based on static assays (Fig. 6B inset). In conclusion TDT curves (and the associated $R$-scripts) are useful for conversion between static and dynamic assessment of tolerance. The quality of model output depends on the quality and 

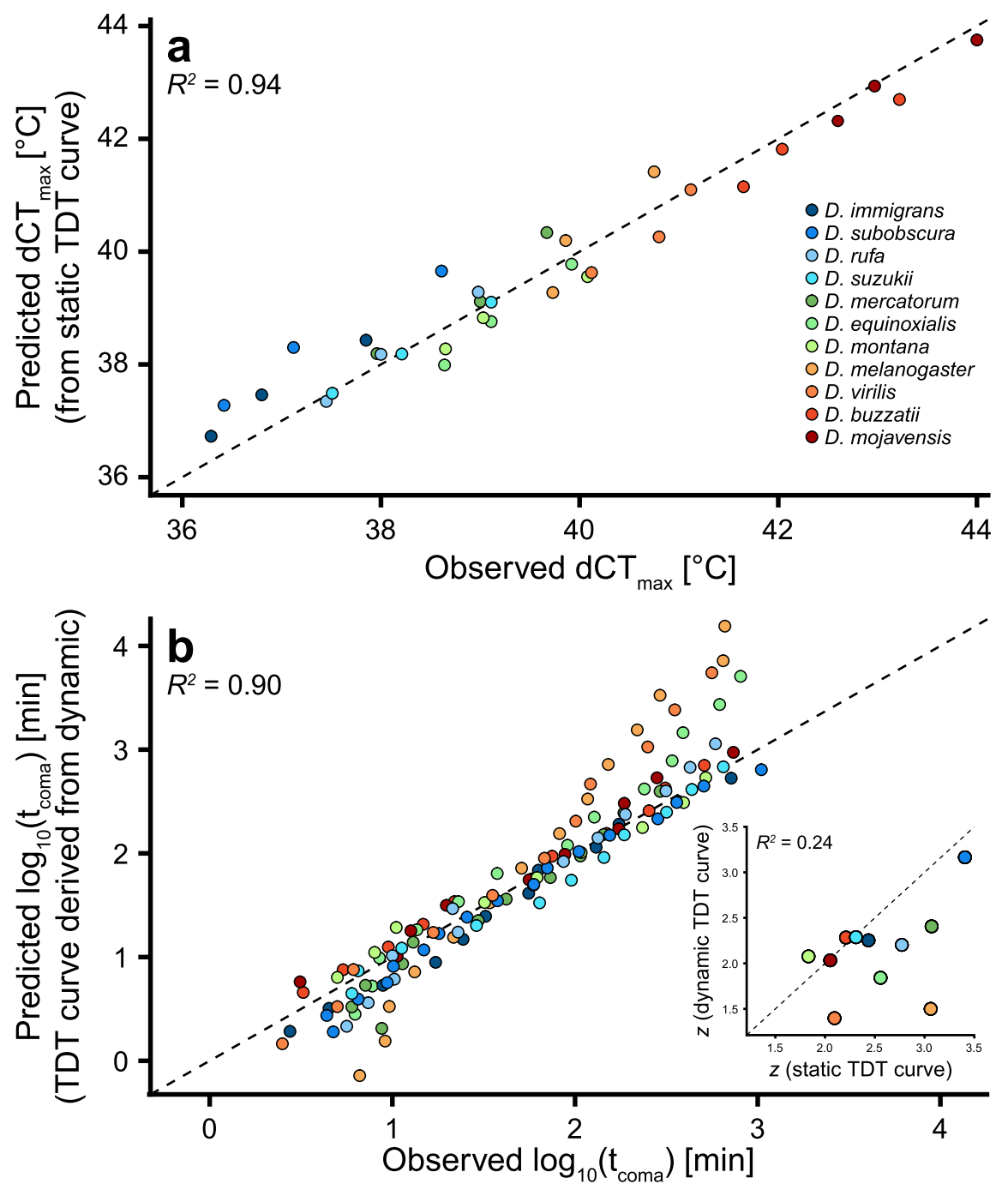

Figure 6. Conversion of heat tolerance measures between static and dynamic assays in Drosophila. Data from ${ }^{43}$. (A) Heat tolerance $\left(\mathrm{dCT}_{\max }, d\right.$ for dynamic assays) plotted against predicted $\mathrm{dCT}_{\max }$ derived from speciesspecific TDT curves created from multiple (9-17) static assays. Data are presented for three different ramping rates $\left(0.05,0.1\right.$ and $\left.0.25^{\circ} \mathrm{C} \mathrm{min}{ }^{-1}\right)$. Note that this graph is adapted from Fig. $4 \mathrm{~b} \mathrm{in}{ }^{15}$. (B) TDT parameters based on $\mathrm{dCT}_{\max }$ from three dynamic tests were used to predict $\mathrm{t}_{\text {coma }}$ in static assays. Each point represents an observed vs. predicted value of species- and temperature-specific $\log _{10}\left(t_{\text {coma }}\right)$. (Inset) Species values of the thermal sensitivity parameter $z$ parameterized from TDT curves based on static assays (x-axis) or dynamic assays (y-axis). The dashed line represents the line of unity in all three panels.

quantity of data used as model input, and in this example the poorer model was parameterized from only three dynamic assays while the stronger model was based on 9-17 static assays (see also "Discussion" section below).

Model application for comparison of published data. Thermal tolerance is important for defining the fundamental niche of animals ${ }^{1,2,4}$ and the current anthropogenic changes in climate has reinvigorated the interest in comparative physiology and ecology of thermal limits in ectotherms. Meta-analyses of ectotherm heat tolerance data have provided important physiological, ecological and evolutionary insights ${ }^{5,44-46}$, but such studies are often challenged with comparison of tolerance estimates obtained through very different methodologies.

Species tolerance is likely influenced by acclimation, age, sex, diet, etc. ${ }^{47}$ and also by the endpoint used (onset of spasms, coma, death, etc. ${ }^{27}$ ). Nevertheless, we expected heat tolerance of a species to be somewhat constrained ${ }^{45}$, so here we tested the model by converting literature data for nine species to a single and speciesspecific estimate of tolerance, $\mathrm{sCT}_{\max (1 \mathrm{~h})}$, the temperature that causes heat failure in $1 \mathrm{~h}$ (Fig. 5). The overwhelming result of this analysis is that TDT parameters are useful to convert static and dynamic heat tolerance measures to a single metric, and accordingly, the TDT model and $R$-scripts presented here have promising applications for large-scale comparative meta-analyses of ectotherm heat tolerance where a single metric allows for qualified direct comparison of results from different publications and experimental backgrounds. While this is an 
intriguing and powerful application, we caution that careful consideration should be put into the limitations of this model (see "Discussion" section below).

Practical considerations and pitfalls for model interpretation. As shown above it is possible to convert and reconcile different types of heat tolerance measures using TDT parameters and these parameters can also be used to model heat stress under fluctuating field conditions. Modelling and discussion of TDT predictions beyond the boundaries of the input data has recently gained traction (see examples in ${ }^{48,49}$ ) but we caution that the potent exponential nature of the TDT curve requires careful consideration as it is both easy and enticing to misuse this model.

Input data. The quality of the model output is dictated by the input used for parameterization. Accordingly, we recommend TDT parameterization using several $(>5)$ static experiments that should cover the time and temperature interval of interest, e.g. temperatures resulting in $\mathrm{t}_{\text {coma }}$ spanning $10 \mathrm{~min}$ to $10 \mathrm{~h}$, thus covering both moderate and intense heat exposure. Such an experimental series can verify TDT curve linearity and allows modelling of temperature impacts across a broad range of temperatures and stress durations ${ }^{13,15,22}$. It is tempting to use only brief static experiments (high temperatures) for TDT parameterization, but in such cases, we recommend that the resulting TDT curve is only used to describe heat injury accumulation under severe heat stress intensities. Thus, the thermal sensitivity factor $z$ represents a very powerful exponential factor (equivalent to $Q_{10}=100$ to 100,$000 ;{ }^{15}$ ) which should ideally be parametrized over a broad temperature range (see below). We also include a script that allows TDT parametrization from multiple ramping experiments and again we recommend a broad span of ramping rates to cover the time/temperature interval of interest. A drawback of ramping experiments is the relatively large proportion of time spent at benign temperatures where there is no appreciable heat injury accumulation. Thus, dynamic experiments can conveniently use starting temperatures that are close to the temperature where injury accumulation rate surpasses injury repair rate (see "Discussion" section of "true" $\mathrm{T}_{\mathrm{c}}$ below, in Supplemental Information and ${ }^{19}$ for other considerations regarding ramp experiments).

A final methodological consideration relates to body-temperature in brief static experiments where the animal will spend a considerable proportion of the experiment in a state of thermal disequilibrium (i.e. it takes time to heat the animal). To avoid this, we recommend direct measurement of body temperature (large animals) or container temperature (small animals), and advise against excessive reliance on data from test temperatures that results in coma in less than $10 \mathrm{~min}$.

Extrapolation. Most studies of ectotherm heat tolerance include only a single measure of heat tolerance which is inadequate to parameterize a TDT curve. However, a TDT curve can still be generated from a single measure of tolerance (static or dynamic) if a value of $z$ is assumed (see Supplemental Information). As $z$ differs within species and between phylogenetic groups (Table $S 1^{15,20}$ ), choosing the appropriate value may be difficult and discrepancies between the 'true' and assumed $z$ represent a problem that should be approached with care. In Fig. 7A we illustrate this point in a constructed example where a single heat tolerance measurement is sampled from a 'true' TDT curve (full line; $\mathrm{t}_{\mathrm{coma}}=40 \mathrm{~min}$ at $37^{\circ} \mathrm{C}$ ). Along with this 'true' TDT curve we depict the consequences for model predictions if the assumed value of $z$ is misestimated by $\pm 50 \%$. Extrapolation from the original data point is necessary if an estimate of the temperature that causes coma after $1 \mathrm{~h}$ is desired, however due to limited extrapolation (from 40 to $60 \mathrm{~min}$ ), estimation of $\mathrm{sCT}_{\max (1 \mathrm{~h})}$ values based on the 'true' and $z \pm 50 \%$ are not very different $\left(< \pm 0.22{ }^{\circ} \mathrm{C}\right.$ in this example). Accordingly, moderate extrapolations are associated with minor latent errors and such assumptions were the basis for many data points in our comparative analysis (Fig. 5). If extensive extrapolation is used (here 40 -fold from $40 \mathrm{~min}$ to either 1 or $1600 \mathrm{~min}$, Fig. 7A), the assumed $z$ results in $\mathrm{sCT}_{\max }$ estimates varying $\pm 2{ }^{\circ} \mathrm{C}$ from the true value and even more dramatic discrepancies are seen if $t_{\text {coma }}$ is calculated for the temperatures resulting in the 'true' $\mathrm{sCT}_{\max }$ for $1 \mathrm{~min}$ or $1600 \mathrm{~min}\left(41\right.$ and $33^{\circ} \mathrm{C}$, respectively, table in Fig. 7A). Due to the powerful exponential nature of the TDT curve, extrapolation to 41 or $33^{\circ} \mathrm{C}$ with values of $z \pm 50 \%$ gives predicted $\mathrm{t}_{\text {coma }}$ of $1.5 \mathrm{~s}-3.42 \mathrm{~min}$ ('true' $\mathrm{t}_{\text {coma }}=1 \mathrm{~min}$ ) and $8 \mathrm{~h}-44$ days ('true' $\mathrm{t}_{\text {coma }} \sim 1$ day). Accordingly, excessive extrapolation of TDT curves should be avoided as even moderate errors in the estimate of $z$ can result in dramatic output errors if the TDT curve is extrapolated beyond the domain of the input data.

Breakpoints and incipient lethal temperature. TDT curves are established at critically high temperatures and another cause of concern for extrapolation of TDT curves relates to the boundaries of the model. Below some temperature [incipient lethal temperature $c . f^{13}$, here termed $T_{c}$ (see "Mathematical foundation" section], the processes related to acute heat injury will no longer determine the duration of survival, and graphically this is represented as a breakpoint on the TDT curve (analogous to an Arrhenius breakpoint) (Fig. 7B). The premise of the TDT curve is not valid below $T_{c}$ and hence other processes will limit survival below this temperature resulting in a breakpoint. For most species $T_{c}$ is unknown, and it is possible that this value (breakpoint) will also depend on other factors (acclimation, age, sex, diet, etc.)(compare Fundulus and Drosophila, Fig. 7B). Accordingly, extrapolation beyond the parameterized time-temperature domain of the TDT curve should be met with great caution for instance when modelling over diurnal temperature fluctuations as this likely includes temperatures below the incipient temperature.

The role of repair and acclimation. From the present study and historical data ${ }^{13,14,16,22,25}$ it is clear that damage attained within the boundaries of the TDT curve is additive and that this model can be used to assess heat injury accumulation during fluctuations. Additivity is, however, only empirically validated within the boundaries of the TDT curve (i.e. above $T_{c}$; Fig. $7 \mathrm{~B}$ ), and at temperatures below $T_{c}$ it is likely that heat injury can be repaired (Fig. 7C). A study using split-dose heat exposures interspaced by benign temperature exposure found that breaks 

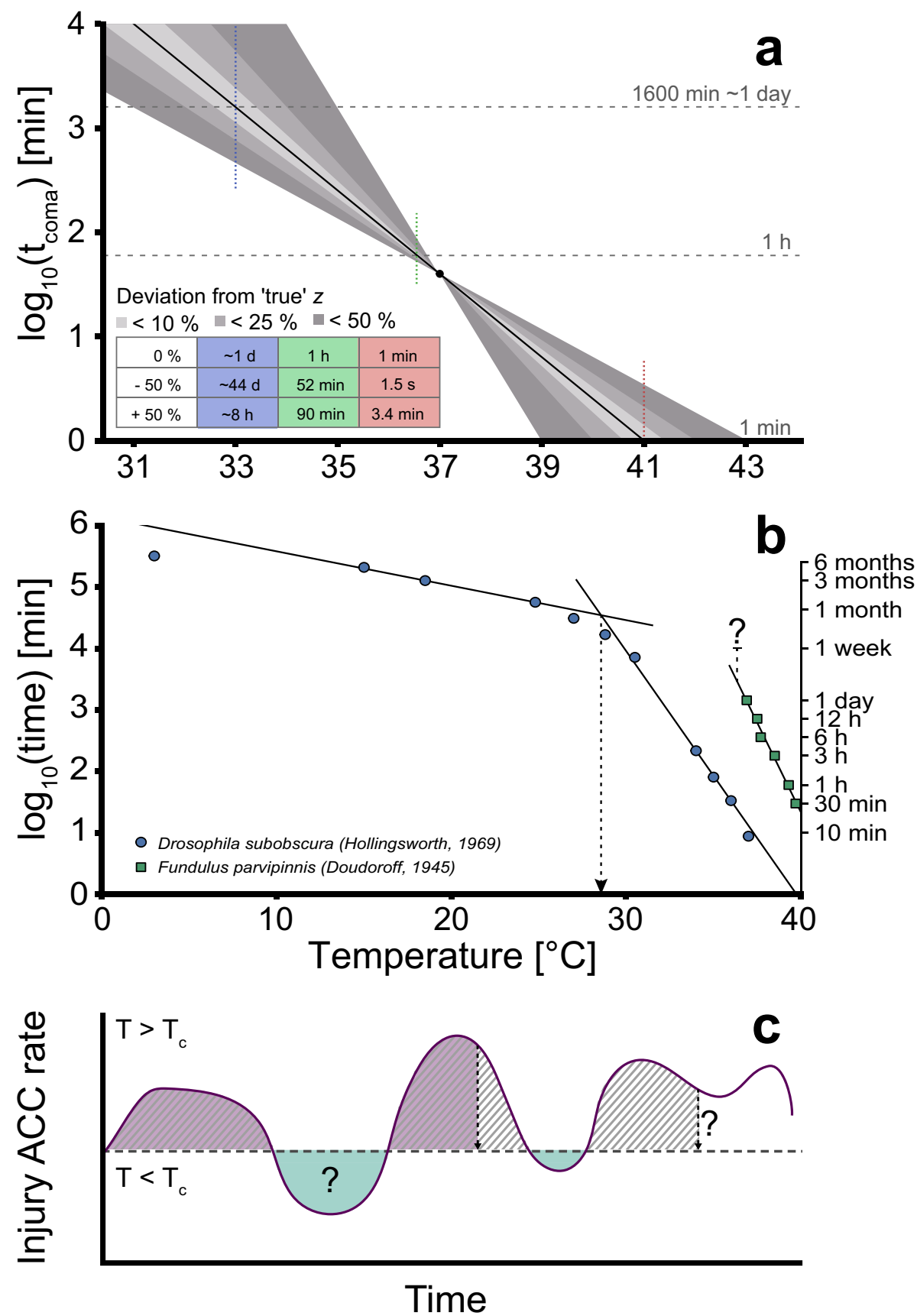

Figure 7. Potential pitfalls of extrapolation and the ambiguity of heat damage repair and hardening. (A) A theoretical TDT curve created from a single point $\left(37^{\circ} \mathrm{C}, 40 \mathrm{~min}\right.$ ) with an assumed 'true' value of $z$ (black line). Grey areas show the TDT curve produced from the same point with deviations from the 'true' $z$ of $\pm 10-50 \%$. Horizontal lines are used to compare estimates of $\mathrm{sCT}_{\max }$ for $1 \mathrm{~min}, 1 \mathrm{~h}$ and $1600 \mathrm{~min}$, while the vertical coloured lines are used to compare time estimates for the temperature of the $\mathrm{sCT}_{\max }$ for the 'true' TDT curve (calculated times in table) (B) The linearity of TDT curves should only be assumed within the time-temperature domain where it is parameterized, and it may vary in temperature and time between species. Data and TDT curve estimates for D. subobscura ${ }^{22}$ and F. parvipinnis ${ }^{25}$. The dashed line for Fundulus represents the temperature with no mortality within the tested time domain ( $\leq 1$ week). The dashed arrow indicates the breakpoint temperature found $b^{22}$. (C) Hypothetical fluctuating temperature profile where temperature (and accordingly the injury accumulation (ACC) rate) fluctuate around the incipient lethal temperature $T_{c}$ (the temperature where injury accumulation rate surpasses injury repair rate, i.e. net injury accumulation). The purple area indicates the part of the temperature profile that would attain the critical amount of injury, under the assumption that no repair or hardening (i.e. processes counteracting injury accumulation) takes place in the green shaded areas. However, when little is known about the processes counteracting injury accumulation and their relation to temperature, it is difficult to predict when coma onset occurs (hatched area). 
$\left(>6 \mathrm{~h}\right.$ ) between heat exposure disrupted additivity, suggesting that injury is repaired at benign temperature ${ }^{50}$. Injury repair rate is largely understudied but repair rate is generally increasing with temperature ${ }^{51-53}$. It is therefore an intriguing and promising idea to include a temperature-dependent repair function in more advanced modelling of heat injury. Until such repair processes are introduced in the model, we recommend that additivity of heat injury is evaluated critically if it involves periods at temperatures both above and below $T_{c}$ (i.e. over consecutive days, see $\mathrm{also}^{13}$ ). An alternative, but not mutually excluding, explanation of increased heat resilience in split-dose experiments relates to the contribution of heat hardening as it is likely that the first heat exposure in a series can induce hardening responses that increase resilience (and thus change the TDT parameters) when a second heat exposure occurs. Such issues of repeated thermal stress have been discussed previously ${ }^{54}$ but for the purpose of the present study the main conclusion is that simple TDT curve modelling is not applicable to fluctuations bracketing $T_{c}$ unless this is empirically validated. Future studies could address this issue as inclusion of repair functions would add further promise to the use of TDT curves in modelling of the impacts of temperature fluctuations.

Received: 20 March 2021; Accepted: 2 June 2021

Published online: 18 June 2021

\section{References}

1. Addo-Bediako, A., Chown, S. L. \& Gaston, K. J. Thermal tolerance, climatic variability and latitude. Proc. Biol. Sci. 267, 739-745 (2000).

2. Angilletta, M. J. Thermal Adaptation: A Theoretical and Empirical Synthesis. (Oxford University Press, 2009). https://doi.org/10. 1093/acprof:oso/9780198570875.001.1.

3. Cossins, A. R. \& Bowler, K. Temperature Biology of Animals (Chapman and Hall, 1987). https://doi.org/10.1007/978-94-009-3127-5.

4. Sunday, J. M., Bates, A. E. \& Dulvy, N. K. Global analysis of thermal tolerance and latitude in ectotherms. Proc. R. Soc. B Biol. Sci. 278, 1823-1830 (2011)

5. Sunday, J. M., Bates, A. E. \& Dulvy, N. K. Thermal tolerance and the global redistribution of animals. Nat. Clim. Change 2, 686-690 (2012).

6. Bigelow, W. D. The logarithmic nature of thermal death time curves. J. Infect. Dis. 29, 528-536 (1921).

7. Santos, M., Castañeda, L. E. \& Rezende, E. L. Making sense of heat tolerance estimates in ectotherms: lessons from Drosophila. Funct. Ecol. 25, 1169-1180 (2011).

8. Sinclair, B. J., Alvarado, L. E. C. \& Ferguson, L. V. An invitation to measure insect cold tolerance: methods, approaches, and workflow. J. Therm. Biol. 53, 180-197 (2015).

9. Terblanche, J. S., Deere, J. A., Clusella-Trullas, S., Janion, C. \& Chown, S. L. Critical thermal limits depend on methodological context. Proc. R. Soc. B Biol. Sci. 274, 2935-2942 (2007).

10. Terblanche, J. S. et al. Ecologically relevant measures of tolerance to potentially lethal temperatures. J. Exp. Biol. 214, 3713-3725 (2011).

11. Andersen, J. L. et al. How to assess Drosophila cold tolerance: chill coma temperature and lower lethal temperature are the best predictors of cold distribution limits. Funct. Ecol. 29, 55-65 (2015).

12. Chown, S. L., Jumbam, K. R., Sørensen, J. G. \& Terblanche, J. S. Phenotypic variance, plasticity and heritability estimates of critical thermal limits depend on methodological context. Funct. Ecol. 23, 133-140 (2009).

13. Fry, F. E. J., Hart, J. S. \& Walker, K. F. Lethal temperature relations for a sample of young speckled trout, Salvelinus fontinalis. Univ. Toronto Stud. Biol. Ser. 54. Publ. Ontario Fish. Res. Lab. 66, 9-35 (1946).

14. Jacobs, M. H. Acclimation as a factor affecting the upper thermal death points of organisms. J. Exp. Zool 27, 427-442 (1919).

15. Jørgensen, L. B., Malte, H. \& Overgaard, J. How to assess Drosophila heat tolerance: unifying static and dynamic tolerance assays to predict heat distribution limits. Funct. Ecol. 33, 629-642 (2019).

16. Kilgour, D. M. \& McCauley, R. W. Reconciling the two methods of measuring upper lethal temperatures in fishes. Environ. Biol. Fishes 17, 281-290 (1986).

17. Kingsolver, J. G. \& Umbanhowar, J. The analysis and interpretation of critical temperatures. J. Exp. Biol. 221, jeb167858 (2018).

18. Kovacevic, A., Latombe, G. \& Chown, S. L. Rate dynamics of ectotherm responses to thermal stress. Proc. R. Soc. B Biol. Sci. 286, 20190174 (2019).

19. Overgaard, J., Kristensen, T. N. \& Sørensen, J. G. Validity of thermal ramping assays used to assess thermal tolerance in Arthropods. PLOS ONE 7, e32758 (2012).

20. Rezende, E. L., Castañeda, L. E. \& Santos, M. Tolerance landscapes in thermal ecology. Funct. Ecol. 28, 799-809 (2014).

21. Bowler, K. A study of the factors involved in acclimatization to temperature and death at high temperatures in Astacus pallipes I. Experiments on intact animals. J. Cell. Comp. Physiol. 62, 119-132 (1963).

22. Hollingsworth, M. J. Temperature and length of life in Drosophila. Exp. Gerontol. 4, 49-55 (1969).

23. Maynard Smith, J. Temperature tolerance and acclimatization in Drosophila subobscura. J. Exp. Biol. 34, 85-96 (1957).

24. Schmidt-Nielsen, K. Animal Physiology Adaptation and Environment (Cambridge University Press, 1997).

25. Doudoroff, P. The resistance and acclimatization of marine fishes to temperature changes. II. Experiments with fundulus and atherinops. Biol. Bull. 88, 194-206 (1945).

26. Brett, J. R. Some principles in the thermal requirements of fishes. Q. Rev. Biol. 31, 75-87 (1956).

27. Lutterschmidt, W. I. \& Hutchison, V. H. The critical thermal maximum: data to support the onset of spasms as the definitive end point. Can. J. Zool. 75, 1553-1560 (1997).

28. Lutterschmidt, W. I. \& Hutchison, V. H. The critical thermal maximum: history and critique. Can. J. Zool. 75, 1561-1574 (1997).

29. Mellanby, K. Acclimatization and the thermal death point in insects. Nature 173, 582 (1954).

30. Larsen, E. B. Problems of heat death and heat injury. Experiments on some species of Diptera. Kgl. Danske Vidensk Selsk. Biol. Medd. 19, 1-52 (1943).

31. O'Sullivan, J. D. B., MacMillan, H. A. \& Overgaard, J. Heat stress is associated with disruption of ion balance in the migratory locust, Locusta migratoria. J. Therm. Biol. 68, 177-185 (2017).

32. MacMillan, H. A., Nørgård, M., MacLean, H. J., Overgaard, J. \& Williams, C. J. A. A critical test of Drosophila anaesthetics: isoflurane and sevoflurane are benign alternatives to cold and $\mathrm{CO}_{2}$. J. Insect Physiol. 101, 97-106 (2017).

33. Kovacevic, A., Latombe, G. \& Chown, S. L. Rate dynamics of ectotherm responses to thermal stress. Dataset https://doi.org/10. 26180/5c467981f3158 (2019).

34. Bennett, J. M. et al. Data from: GlobTherm, a global database on thermal tolerances for aquatic and terrestrial organisms. Dryad Digit. Repos. https://doi.org/10.1038/sdata.2018.22 (2018). 
35. Cereja, R. Critical thermal maxima in aquatic ectotherms. Ecol. Indic. 119, 106856 (2020).

36. Armstrong, J. W., Tang, J. \& Wang, S. Thermal death kinetics of Mediterranean, Malaysian, melon, and oriental fruit fly (Diptera: Tephritidae) eggs and third instars. J. Econ. Entomol. 102, 522-532 (2009).

37. Neven, L. G. Physiological responses of insects to heat. Postharvest Biol. Technol. 21, 103-111 (2000).

38. Bowler, K. Heat death in poikilotherms: Is there a common cause?. J. Therm. Biol. 76, 77-79 (2018).

39. Marsh, A. C. Microclimatic factors influencing foraging patterns and success of the thermophillic desert ant, Ocymyrmex barbiger. Insectes Soc. 32, 286-296 (1985).

40. Grant, B. W. \& Dunham, A. E. Thermally imposed time constraints on the activity of the desert lizard Sceloporus merriami. Ecology 69, 167-176 (1988).

41. Easterling, D. R. et al. Climate extremes: observations, modeling, and impacts. Science 289, 2068-2074 (2000).

42. Marcy, B. C. Jr. Survival of young fish in the discharge canal of a nuclear power plant. J. Fish. Res. Board Canada 28, 1057-1060 (1971).

43. Jørgensen, L. B., Malte, H. \& Overgaard, J. Data from: How to assess Drosophila heat tolerance: unifying static and dynamic tolerance assays to predict heat distribution limits. Dryad Digit. Repos. https://doi.org/10.5061/dryad.840j728 (2019).

44. Sunday, J. et al. Thermal tolerance patterns across latitude and elevation. Philos. Trans. R. Soc. B Biol. Sci. 374, 20190036 (2019).

45. Araújo, M. B. et al. Heat freezes niche evolution. Ecol. Lett. 16, 1206-1219 (2013).

46. Leiva, F. P., Calosi, P. \& Verberk, W. C. E. P. Scaling of thermal tolerance with body mass and genome size in ectotherms: a comparison between water- and air-breathers. Philos. Trans. R. Soc. B Biol. Sci. 374, 20190035 (2019).

47. Hangartner, S. \& Hoffmann, A. A. Evolutionary potential of multiple measures of upper thermal tolerance in Drosophila melanogaster. Funct. Ecol. 30, 442-452 (2016).

48. Rezende, E. L., Bozinovic, F., Szilágyi, A. \& Santos, M. Predicting temperature mortality and selection in natural Drosophila populations. Science 369, 1242-1245 (2020).

49. Buckley, L. B. Body size shapes thermal stress. Nat. Clim. Change 11, 5-6 (2021).

50. Kashmeery, A. M. S. \& Bowler, K. A study of recovery from heat injury in the blowfly (Callipora erythrocephala) using split-dose experiments. J. Therm. Biol. 2, 183-184 (1977).

51. Bowler, K. \& Kashmeery, A. M. S. Recovery from heat injury in the blowfly, Calliphora erythrocephala. J. Therm. Biol. 4, 197-202 (1979).

52. Iandolo, J. J. \& Ordal, Z. J. Repair of thermal injury of Staphylococcus aureus. J. Bacteriol. 91, 134-142 (1966).

53. Dingley, F. \& Maynard Smith, J. Temperature acclimatization in the absence of protein synthesis in Drosophila subobscura. J. Insect Physiol. 14, 1185-1194 (1968).

54. Colinet, H., Sinclair, B. J., Vernon, P. \& Renault, D. Insects in fluctuating thermal environments. Annu. Rev. Entomol. 60, 123-140 (2015).

\section{Acknowledgements}

The authors would like to thank Kirsten Kromand for help with fly maintenance and John S. Jensen for making the rack for fly testing. The authors thank InEE-CNRS and University of Rennes 1 who support the International Research Project (IRP) 'Phenomic responses of invertebrates to changing environments and multiple stress' (PRICES).

\section{Author contributions}

J.O., H.M. and L.B.J. conceived the ideas and designed the study; H.M. and M.Ø. formulated the mathematical model and prepared $R$ scripts, N.A.K. and L.B.J. collected the data; L.B.J., J.O., H.M., N.A.K. and M.Ø. analysed the data; L.B.J. and J.O. led the writing of the manuscript. All authors contributed critically to the drafts and gave final approval for publication.

\section{Competing interests}

The authors declare no competing interests.

\section{Additional information}

Supplementary Information The online version contains supplementary material available at https://doi.org/ 10.1038/s41598-021-92004-6.

Correspondence and requests for materials should be addressed to L.B.J.

Reprints and permissions information is available at www.nature.com/reprints.

Publisher's note Springer Nature remains neutral with regard to jurisdictional claims in published maps and institutional affiliations.

(c) (i) Open Access This article is licensed under a Creative Commons Attribution 4.0 International License, which permits use, sharing, adaptation, distribution and reproduction in any medium or format, as long as you give appropriate credit to the original author(s) and the source, provide a link to the Creative Commons licence, and indicate if changes were made. The images or other third party material in this article are included in the article's Creative Commons licence, unless indicated otherwise in a credit line to the material. If material is not included in the article's Creative Commons licence and your intended use is not permitted by statutory regulation or exceeds the permitted use, you will need to obtain permission directly from the copyright holder. To view a copy of this licence, visit http://creativecommons.org/licenses/by/4.0/.

(C) The Author(s) 2021 